\title{
Astrometric Programmes of the Abastumani Observatory
}

Soso M. Chanturiya, Raguli I. Inasaridze, Rolan I. Kiladze, \& Omar M. Kurtanidze

Abastumani Astrophysical Observatory, 383762 Abastumani, Republic of Georgia

\begin{abstract}
Astrometric investigations undertaken with the $70 \mathrm{~cm}$ meniscus and $40 \mathrm{~cm}$ double astrograph telescopes are considered. We describe the determination of accurate positions for minor planets, giant-planet satellites, late-type stars, extragalactic radio-sources,optically discovered quasars and compact extragalactic objects in the Second Byurakan Survey.
\end{abstract}

\section{A Few Historical Excursions}

Although the scientific activities at the Abastumani Observatory were concentrated mainly on astrophysics, from time to time astrometrical problems, be it a partial investigation of a telescope, or a determination of the position of an individual ob ject, were also solved. Tevzadze (1940) carried out work in the field of meridional astronomy for the investigation of a pivot transit instrument, but this was not later continued. In Abastumani at different times, precise positions of novae, comets and minor planets have been determined, with an accuracy better than one arcsec. A significant contribution in the field of modern photographic astrometry at Abastumani was made in the early 1960's. Due to the efforts of outstanding astrometrists from the Pulkovo school, such as A. Dache, A. Kiselev and others, young scientists were prepared for conducting astrometric observations of high quality.

In addition, astrometric investigation of the $70 \mathrm{~cm}$ Maksutov system mirror telescope AS-32 ( $\mathrm{f}=2102)$ was done by Khatisashvili. As a result, it was shown that such reflectors could solve a series of astrometric problems (with a high degree of accuracy of $0.1 \mathrm{arcsec}$ ), for a wide field of diameter up to three degrees. Investigation of the $40 \mathrm{~cm}$ double Zeiss astrograph (Khatisashvili, Inasaridze 1986) has shown that a new high-quality astrometric device has been obtained with a field of $5.7^{\circ} \times 5.7^{\circ}$ and a focal distance of $3000 \mathrm{~mm}$.

\section{The Solar System}

\subsection{The Minor Planets}

Khatisashvili has continued observations of minor planets and comets. This study has acquired a more systematic character since 1976, when the Abastu- 
mani Observatory joined in the All-Union Programme. (Kiladze 1977; Khatisashvili et al. 1987). This work is in progress at present.

\subsection{The Giant Planets and their Satellites}

For the first time positional photographic observations of the satellites Phobos and Deimos have been obtained. The standard error for a single observation at outer coincidence (Shor 1976) is $0.5-0.6$ arcsec (Khatisashvili \& Salukvadze 1975).

Kiseleva \& Chanturiya (1987) have continued with the work on positional observations of major planets and their satellites in the framework of the AllUnion programme "ORBITA". So far, Chanturiya has obtained more than one thousand astro-negatives with the $40 \mathrm{~cm}$ telescope of the Abastumani Observatory. The planets Jupiter, Saturn, Uranus and Neptune are being observed, as well as the Gallilean satellites of Jupiter, Saturn's II-VIII satellites, the Uranus III and IV satellites and the Neptune I satellite. By 1998 the astronomers will have acquired a 15-year series of observations characterized by errors of $0.2-0.3$ arcsec. Comparison of the observations with new theories for the motion of planets and their satellites, gives good agreement within one arcsec. Full astrometric processing, comparison of observations with the theory and publication of the results are unlikely to be completed before the end of the century.

Making use of new observational techniques, Chanturiya and Salukvadze carried out a series of observations of Phobos, Deimos and Mars in 1986 and 1988 with the $125 \mathrm{~cm} \mathrm{RC}$ telescope $(\mathrm{f} / 13)$. The results were published with Kalinichenko and Kiseleva (1990). The accuracy of the observations was 0.2-0.3 arcsec.

In 1982-1985 Kiladze (1986a) carried out positional observations of Pluto which have been included in his monograph (1986b). A year prior to the discovery of Charon, Kiladze (1977) predicted the existence of Pluto's satellite in the following phrase: "In conclusion we point out that a host of small particles might have surrounded Pluto as well, which must possess, in acordance with our theory, an unknown satellite, waiting for discovery". It might be noted that Kiladze carried out a few observations before the discovery of Charon but without success due to bad weather.

\section{The Stars}

Accurate optical positions of 42 novae have been measured by Khatisashvili (1971a). Inasaridze joined in the All-Union "Photographic Sky Survey" programme, once suggested by Ukrainian astrometrists. Using the $40 \mathrm{~cm}$ double astrograph, he has already observed the region from $-20^{\circ}$ to $30^{\circ}$ with a limiting B-magnitude of 17 and an envisaged accuracy of 0.1 arcsec for positions and $0.007 \mathrm{arcsec} / \mathrm{yr}$ for proper motions over a time-baseline of 30 years.

Chanturiya $(1980 \mathrm{a} ; 1980 \mathrm{~b} ; 1982)$ has worked on the determination of proper motions of zirconium stars with the aim of obtaining absolute magnitudes of these Red Giant stars using the method of statistical parallaxes. The observations were obtained using the $70 \mathrm{~cm}$ meniscus telescope. The "CARTE DU CIEL" catalogues were used as the first epoch and a proper motion accuracy 
of $0.004 \mathrm{arcsec} / \mathrm{yr}$ was achieved for seventy-four zirconium stars (S-type stars). The space distribution of the S-type stars was studied using the data obtained.

\section{QSO and Radio Sources}

Inasaridze $(1984 ; 1987 ; 1989)$ is participating in the "CONFOR" (Connection of Frames in Optics and Radio) programme. This programme aims to link the optical counterparts of compact extragalactic radio sources with the fundamental coordinate system. He has published optical positions of forty-two radio sources. At present, sixteen additional objects have been measured. To avoid determining the coordinates of intermediate reference stars, Insaridze used a method proposed by Prof. Kiladze. To exclude the brightness equation, a so-called "chromium light filter" is used. This covers the field including the reference stars while the central part with the radio sources remains clear. Application of this method strongly reduces the position errors as well as the number of measurements needed.

Kurtanidze (1989) began a programme at Abustamani to determine optical positions of QSOs found in optical surveys; namely in the Second Byurakan Survey (Markarian et al. 1983). Fields are observed if they include at least ten quasars and a dozen compact extragalactic objects. To reduce the large brightness differences between QSOs $\left(m_{B}=17-19\right)$ and reference stars $\left(m_{B}=8-11\right)$ he also used the chromium filter described above, with a reduced transmission for the reference stars, so that the densities of the reference stars on the plate are the same.

\section{Future Prospects}

In the near future the full treatment of observations of giant planets and their satellites is considered to be rather important. Precise optical positions will be determined for the radio sources in the list proposed by the CONFOR international programme and for QSOs and compact extra- galactic objects in the Second Byurakan Sky survey. Positions will be obtained using the $70 \mathrm{~cm}$ meniscus telescope .

\section{References}

Chanturiya S. M., 1980a, Bull. Abast. Astrophys. Observ., 53, 77

Chanturiya S. M., 1980b, Bull. Abast. Astrophys. Observ., 53, 96

Chanturiya S. M., 1982, Bull. Abast. Astrophys. Observ., 55, 152

Inasaridze R. Ia., 1984, Astron. Circular, 1309, 6

Inasaridze R. Ia., 1987, Bull. Abast. Astrophys. Observ., 62, 132

Inasaridze R. Ia., 1989, Bull. Abast. Astrophys. Observ., 68, 191

Kalinichenko O. A, Kiseleva T. P., Salukvadze G. N. \& Chanturiya S. M., 1990, Bull. Abast. Astrophys. Observ., 68, 99

Khatisashvili A. Sh. \& Salukvadze G. N., 1975, Bull. Abast. Astrophys. Observ., 46,217 
Khatisashvili A. Sh. \& Inasaridze R. Ia., 1986, Bull. Abast. Astrophys. Observ., 61,289

Khatisashvili A. Sh. \& Inasaridze R. Ia., 1987, Bull. Abast. Astrophys. Observ., 62,131

Kiladze R. I., 1977, Bull. Abast. Astrophys. Observ., 48, 211

Kiladze R. I., 1986a, Bull. Inst. Theoretical Astron., 15, 602

Kiladze R. I., 1986b, Sovremennoe Vrashenie Planet, (Mecniereba, Tblisi)

Kiselev A. A. \& Chanturiya S. M., 1987, Bull. Abast. Astrophys. Observ., 62, 118

Kurtanidze O. M., 1989, unpublished

Markarian B., Lipovetsky V. \& Stepanian J. 1979, Astrophysics, 19, 29

Shor V. A., 1976, Trudy Inst. Theoretical Astron., 15, 103

Tevzadze G. A., 1940, Bull. Abast. Astrophys. Observ., 5, 49 